\title{
Room for New Physics in the Rayleigh-Jeans Tail of the Cosmic Microwave Background
}

\author{
Maxim Pospelov, ${ }^{1,2,3}$ Josef Pradler, ${ }^{4}$ Joshua T. Ruderman, ${ }^{5,3}$ and Alfredo Urbano ${ }^{6,3}$ \\ ${ }^{1}$ Perimeter Institute for Theoretical Physics, 31 Caroline Street North, Waterloo, Ontario N2L 2Y5, Canada \\ ${ }^{2}$ Department of Physics and Astronomy, University of Victoria, Victoria, British Columbia V8P 5C2, Canada \\ ${ }^{3}$ Theoretical Physics Department, CERN, CH-1211 Geneva 23, Switzerland \\ ${ }^{4}$ Institute of High Energy Physics, Austrian Academy of Sciences, Nikolsdorfergasse 18, 1050 Vienna, Austria \\ ${ }^{5}$ Center for Cosmology and Particle Physics, Department of Physics, New York University, \\ New York, New York 10003, USA \\ ${ }^{6}$ INFN, sezione di Trieste, SISSA, via Bonomea 265, 34136 Trieste, Italy
}

(Received 29 March 2018; revised manuscript received 5 June 2018; published 20 July 2018)

\begin{abstract}
We show that, despite stringent constraints on the shape of the main part of the cosmic microwave background (CMB) spectrum, there is considerable room for its modification within its Rayleigh-Jeans (RJ) end, $\omega \ll T_{\mathrm{CMB}}$. We construct explicit new physics models that give an order one (or larger) increase of photon count in the RJ tail, which can be tested by existing and upcoming experiments aiming to detect the cosmological $21 \mathrm{~cm}$ emission or absorption signal. This class of models stipulates the decay of unstable particles to dark photons $A^{\prime}$ that have a small mass, $m_{A^{\prime}} \sim 10^{-14}-10^{-9} \mathrm{eV}$, nonvanishing mixing angle $\epsilon$ with electromagnetism, and energies much smaller than $T_{\mathrm{CMB}}$. The nonthermal number density of dark photons can be many orders of magnitude above the number density of CMB photons, and even a small probability of $A^{\prime} \rightarrow A$ oscillations, for values as small as $\epsilon \sim 10^{-9}$, can significantly increase the number of RJ photons. In particular, we show that resonant oscillations of dark photons into regular photons in the interval of redshifts $20<z<1700$ can be invoked as an explanation of the recent tentative observation of a stronger-than-expected absorption signal of $21 \mathrm{~cm}$ photons. We present a model that realizes this possibility, where meV mass dark matter decays to dark photons, with a lifetime longer than the age of the Universe.
\end{abstract}

DOI: 10.1103/PhysRevLett.121.031103

Introduction.-Modern cosmology owes much of its advance to precision observations of the cosmic microwave background (CMB). Both the spectrum of the CMB and its angular anisotropies are precisely measured by a number of landmark experiments [1-3]. CMB physics continues its advance [4] into probing both the standard $\Lambda \mathrm{CDM}$ model to higher precision and possible new physics that can manifest itself as small deviations from theoretical expectations. In addition, a qualitatively new cosmological probe, the physics of $21 \mathrm{~cm}$ emission or absorption at the end of the "dark ages," may come into play in the very near future [5].

Cosmology has been a vital tool for learning about physics beyond the standard model (SM). About onequarter of our Universe's energy budget is comprised of cold dark matter (DM). The precision tools of cosmology provide serious constraints on the properties of DM, which instead of coming "alone," may be a part of an extended

Published by the American Physical Society under the terms of the Creative Commons Attribution 4.0 International license. Further distribution of this work must maintain attribution to the author(s) and the published article's title, journal citation, and DOI. Funded by SCOAP. dark sector, comprising new matter and radiation fields, and new forces. Recent years have seen a significant increase in studies of dark sectors, both in connection with terrestrial experiments and in cosmology [6-8].

If such light fields are thermally excited, they can be detected through their gravitational interaction alone, as they would modify the Hubble expansion rate, affect the outcome of big bang nucleosynthesis, and modify the angular anisotropies of the CMB. The resulting constraint is phrased in terms of the number of effective neutrino degrees of freedom which, according to the latest observational bounds, $N_{\text {eff }}=3.04 \pm 0.33$ [3] (95\% C.L.), is consistent with the expectations from standard cosmology. Nonthermal dark radiation (DR) is considered in the literature less often, although many dark sector processes may lead to its appearance.

In recent papers $[9,10]$, interacting DR was examined in the regime where the individual quanta are fewer in number but harder in energy than typical CMB photons, $n_{\mathrm{DR}} \ll$ $n_{\mathrm{CMB}}$ and $\omega_{\mathrm{DR}} \gg \omega_{\mathrm{CMB}}$, but such that the $N_{\text {eff }}$ constraint is satisfied. In this Letter, we study the alternative, DR quanta much softer, but more numerous than CMB photons,

$\omega_{\mathrm{DR}} \ll \omega_{\mathrm{CMB}}, \quad n_{\mathrm{DR}}>n_{\mathrm{RJ}}, \quad \omega_{\mathrm{DR}} n_{\mathrm{DR}} \ll \rho_{\mathrm{tot}}$, 
where $\rho_{\text {tot }}$ is the total energy density of radiation and DM, $n_{\mathrm{DR}}$ is the number density of DR quanta, and $n_{\mathrm{RJ}}$ represents the low-energy Rayleigh-Jeans (RJ) tail of the standard CMB Planck distribution, $n_{\mathrm{RJ}} \approx T \omega_{\max }^{2} /\left(2 \pi^{2}\right) \approx 0.21 x_{\max }^{2} n_{\mathrm{CMB}}$, where we use units $\hbar=c=k=1$, and where we define the normalized photon energy, $x \equiv \omega / T_{\mathrm{CMB}} \cdot n_{\mathrm{CMB}}=$ $2 \zeta(3) / \pi^{2} T_{\mathrm{CMB}}^{3} \simeq 0.24 T_{\mathrm{CMB}}^{3}$ is the full Planckian number density and $x_{\max }=\omega_{\max } / T_{\mathrm{CMB}}$ is a (somewhat arbitrary) maximum frequency of the low-energy RJ interval, $x_{\max } \ll 1$. If, for example, we take $x_{\max }=2 \times 10^{-3}$, then we find $n_{\mathrm{RJ}} / n_{\mathrm{CMB}} \simeq 10^{-6}$.

The number of DR quanta may significantly exceed $n_{\mathrm{RJ}}$. Saturating the constraint on $N_{\text {eff }}$ for the DR that matches the CMB frequencies with $x_{\max } \sim 2 \times 10^{-3}$, we find $n_{\mathrm{DR}} \leq$ $1.5 \times 10^{2} n_{\mathrm{CMB}}$. This is because the energy density of a DR component can be written as $\rho_{\mathrm{DR}}=\omega_{\mathrm{DR}} n_{\mathrm{DR}}=$ $\Delta N_{\text {eff }} \frac{7}{8}\left(\frac{4}{11}\right)^{4 / 3} \rho_{\mathrm{CMB}}$, where we separated the SM contribution $N_{\text {eff }}=N_{\text {eff }}^{\mathrm{SM}}+\Delta N_{\text {eff }}$ with $N_{\text {eff }}^{\mathrm{SM}}=3.046$. Alternatively, letting $5 \%$ of the DM energy density $[11,12]$ convert to DR in the same frequency range after $\mathrm{CMB}$ decoupling, $n_{\mathrm{DR}} \leq 3.3 \times 10^{5} n_{\mathrm{CMB}}$. Thus, soft DR quanta can outnumber the RJ CMB photons by up to 11 orders of magnitude.

What are the observational consequences of such soft and numerous DR quanta? Very light fields often have their interactions enhanced (suppressed) at high (low) energies. This is the case for neutrinos that have Fermi-type interactions with atomic constituents, as well as for axions that have effective interactions with fermions and gauge bosons. This type of DR would be very difficult to see directly. There is, however, one class of new fields that can manifest their interactions at low energies and low densities. These are light vector particles (often called dark photons) $A^{\prime}$ that develop a mixing with ordinary photons $\epsilon F_{\mu \nu}^{\prime} F_{\mu \nu}$ [13]. The apparent number count of CMB radiation can be modified by photon or dark photon oscillation

$$
\frac{d n_{A}}{d \omega} \rightarrow \frac{d n_{A}}{d \omega} \times P_{A \rightarrow A}+\frac{d n_{A^{\prime}}}{d \omega} \times P_{A^{\prime} \rightarrow A},
$$

where $P_{A \rightarrow A}=1-P_{A \rightarrow A^{\prime}}$ is the photon survival probability, while $P_{A^{\prime} \rightarrow A}$ is the probability of $A^{\prime} \rightarrow A$ conversion. Previous constraints from $A \rightarrow A^{\prime}$ were derived [14,15] using COBE-FIRAS data [16]. The point of the present Letter is that the RJ tail of the CMB can get a significant boost due to the second term in (2) without contradicting FIRAS. The physics of the $21 \mathrm{~cm}$ line provides a useful tool to probe DR through the apparent modification of the lowenergy tail of the CMB. Previous studies [17-19] have considered $A^{\prime} \rightarrow A$ when $A^{\prime}$ is cold DM instead of DR.

The EDGES experiment recently presented a tentative detection of $21 \mathrm{~cm}$ absorption coming from the interval of redshifts $z=15-20$ [20]. The strength of the absorption signal is expected to be proportional to $1-T_{\mathrm{CMB}} / T_{s}$ [21], where $T_{\mathrm{CMB}}$ counts the number of CMB photons interacting with the two-level hydrogen hyperfine system, and $T_{s}$
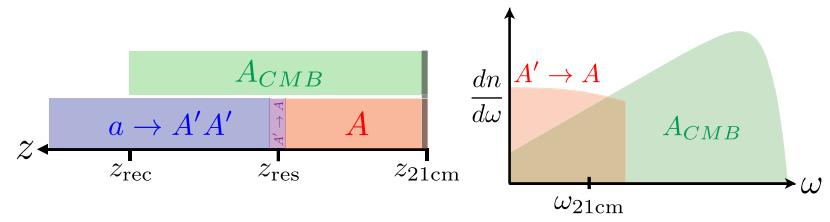

FIG. 1. Dark matter $a$ decays to soft massive dark photons $A^{\prime}$, which oscillate into ordinary photons $A$. The conversion happens resonantly at redshift $z_{\text {res }}$ before the formation of the $21 \mathrm{~cm}$ absorption signal. The converted photons (red) add to the CMB photon count (green) in the RJ tail.

is the spin temperature. The relevant photons have energy $\omega_{0}=5.9 \mu \mathrm{eV}$ at redshift $z \approx 17$, and therefore reside deep within the RJ tail, $x \approx 1.4 \times 10^{-3}$. This is much lower energy than direct measurements from FIRAS, at $x>1.2$ [16], and ARCADE 2, which probes as low as $x=0.056$ [22]. Earlier measurements constrain $x \sim 0.02-0.04$ with larger uncertainties $[23,24]$.

The locations of the left or right boundaries of the claimed EDGES signal agree with standard cosmological expectations, but the amount of absorption seems to indicate a more negative $1-T_{\mathrm{CMB}} / T_{s}$ temperature contrast than expected. Given that the spin temperature $T_{s}$ cannot drop below the baryon temperature $T_{b}$, a naive interpretation of this result could consist in lower-than-expected $T_{b}$ or higher $T_{\mathrm{CMB}}$. Together with related prior work [25,26], a number of possible models were suggested [27-31], which typically have difficulty passing other constraints [32-37]. Our mechanism, the oscillation of nonthermal DR into visible photons (illustrated in Fig. 1), can easily accommodate EDGES consistent with other constraints. In the remainder of this Letter, we provide more details on the suggested mechanism and identify the region of parameter space where $21 \mathrm{~cm}$ physics can provide the most sensitive probe of DR.

Decay of unstable relics into dark radiation.-The framework described in the Introduction allows for significant flexibility with respect to the actual source of nonthermal soft DR. To give a concrete realization of the proposed mechanism, we specify a model of unstable scalar particles $a$ that couple to dark photons via an effective dimension five operator,

$$
\mathcal{L}=\frac{1}{2}\left(\partial_{\mu} a\right)^{2}-\frac{m_{a}^{2}}{2} a^{2}+\frac{a}{4 f_{a}} F_{\mu \nu}^{\prime} \tilde{F}^{\prime \mu \nu}+\mathcal{L}_{A A^{\prime}},
$$

where $\tilde{F}_{\mu \nu}^{\prime}=\frac{1}{2} \epsilon^{\mu \nu \rho \sigma} F_{\rho \sigma}$, and the last term describes the photon-dark photon Lagrangian with corresponding mass and mixing terms for $A^{\prime}$,

$\mathcal{L}_{A A^{\prime}}=-\frac{1}{4} F_{\mu \nu}^{2}-\frac{1}{4}\left(F_{\mu \nu}^{\prime}\right)^{2}-\frac{\epsilon}{2} F_{\mu \nu} F_{\mu \nu}^{\prime}+\frac{1}{2} m_{A^{\prime}}^{2}\left(A_{\mu}^{\prime}\right)^{2}$.

The cosmology of $a$ is model dependent. To keep our discussion general, we assume that an initial relic abundance 

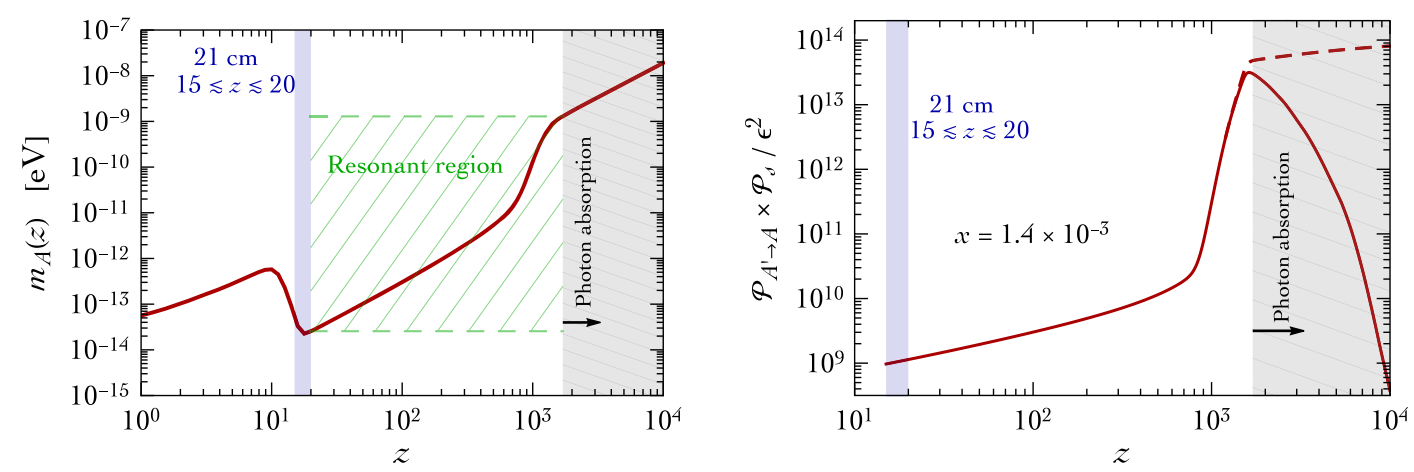

FIG. 2. (Left) Effective photon mass $m_{A}$ as a function of redshift. When resonant oscillation $\left[m_{A}(z)=m_{A^{\prime}}\right]$ occurs between redshift $20 \leq z \lesssim 1700$ (green) it impacts the $21 \mathrm{~cm}$ absorption signal. (Right) Effective conversion probability as a function of redshift. We consider photons with energy $x=\omega / T_{\mathrm{CMB}}=1.4 \times 10^{-3}$, which implies a wavelength of $21 \mathrm{~cm}$ at $z=17$.

of $a$ is present, but we do not specify the method of nonthermal production, which could be misalignment or decay of cosmic strings.

The decay rate of $a \rightarrow 2 A^{\prime}$ into two (transverse) dark photons $A^{\prime}$ is given by

$\Gamma_{a}=\frac{m_{a}^{3}}{64 \pi f_{a}^{2}}=\frac{3 \times 10^{-4}}{\tau_{U}}\left(\frac{m_{a}}{10^{-4} \mathrm{eV}}\right)^{3}\left(\frac{100 \mathrm{GeV}}{f_{a}}\right)^{2}$

The lifetime $\tau_{a}=1 / \Gamma_{a}$ can be either much longer or much shorter than the present age of the Universe, $\tau_{U} \approx$ $13.8 \times 10^{9} \mathrm{yr}$. In the case of short lifetimes, $\tau_{a} \ll \tau_{U}$, the mass of $a$ should be chosen such that the energy of the resulting $A^{\prime}$ matches the $\mathrm{CMB}$ energy in the $\mathrm{RJ}$ tail at the time of decay. We leave detailed study of this possibility for future work and instead focus on the case of a cosmologically long-lived particle $\tau_{a} \gg \tau_{U}$, which is attractive because $a$ can naturally serve as DMIf the mass of $a$ falls in the range $10^{-5}<m_{a}<10^{-1} \mathrm{eV}$, its decay can create significant modifications to the RJ tail of the CMB spectrum via $A^{\prime} \rightarrow A$ oscillations. It is worth noting that this overlaps with the mass range often invoked for axion DM.

$A^{\prime} \leftrightarrow A$ oscillation and constraints.-All constraints on parameters of (3) and (4) can be divided into two groups: those that decouple as $m_{A^{\prime}} \rightarrow 0$ and those that persist in the limit of a massless dark photon. The stellar energy loss constraint due to $A^{\prime} a$ pair production is in this second category, as the in-medium transverse modes of photons can decay via $A_{T}^{*} \rightarrow A^{\prime} a$ even in the $m_{A^{\prime}} \rightarrow 0$ limit. The approximate emission rate is $Q_{A^{*} \rightarrow A^{\prime} a}=\epsilon^{2} m_{A}^{4} n_{T} /\left(96 \pi f_{a}^{2}\right)$, where $n_{T}$ is the number density of transverse plasmons (photons) and $m_{A}$ is the plasma frequency, $m_{A}^{2}=4 \pi \alpha n_{e} / m_{e}$. We recast the stellar bound on emission of Dirac neutrinos due to their magnetic moment [38-40] to obtain the following bound: $\epsilon \times f_{a}^{-1}<2 \times 10^{-9} \times \mathrm{GeV}^{-1}$. The process $e^{+} e^{-} \rightarrow a A^{\prime}$ can lead to thermal populations of $a$ and $A^{\prime}$ in the early Universe. However, the stellar constraint implies that this reaction drops out of equilibrium at temperatures above the electroweak scale, and therefore does not lead to an additional constraint from $N_{\text {eff }}$.

The $\epsilon$ parameter is also constrained via $A \rightarrow A^{\prime}$ oscillations [41], and the resulting bound depends sensitively on $m_{A^{\prime}}$. Stellar energy losses via these oscillations are important only for the higher mass range, $m_{A^{\prime}}>10^{-5} \mathrm{eV}$, as the emission is suppressed by $m_{A^{\prime}}^{2} / m_{A}^{2} \ll 1$ inside stars $[42,43]$. Cosmological $A \leftrightarrow A^{\prime}$ oscillations may be significant if the resonant condition is met, $m_{A^{\prime}}=m_{A}(z)$, where $m_{A}(z)$ is the plasma mass of photons at redshift $z$ $[14,15]$. In the course of cosmological evolution $m_{A}(z) \simeq$ $1.7 \times 10^{-14} \mathrm{eV} \times(1+z)^{3 / 2} X_{e}^{1 / 2}(z)$ scans many orders of magnitude; $X_{e}$ is the free electron fraction that we take from [15]. For any $m_{A^{\prime}}$ in the range $10^{-14}-10^{-9} \mathrm{eV}$, the resonance happens at some redshift $z_{\text {res }}$ corresponding to cosmic time $t_{\text {res }}$; see the left panel of Fig. 2. The resonance ensures that the probability of oscillation is much larger than the vacuum value of $\epsilon^{2}$. Following [14,44],

$$
P_{A \rightarrow A^{\prime}}=P_{A^{\prime} \rightarrow A}=\frac{\pi \epsilon^{2} m_{A^{\prime}}^{2}}{\omega} \times\left|\frac{d \log m_{A}^{2}}{d t}\right|_{t=t_{\mathrm{res}}}^{-1} .
$$

This expression is valid in the limit $P_{A^{\prime} \rightarrow A} \ll 1$; when the probability saturates, we use the full expression of [45]. The probability of oscillation for RJ photons with $x \sim 10^{-3}$ can be $10^{3}$ times larger than for typical CMB photons with $x \sim 1$, because of the $\omega^{-1}$ scaling. The redshift dependence of (6) is shown in the right panel of Fig. 2, assuming $x_{0}=1.4 \times 10^{-3}$. We multiply the conversion probability times the photon survival probability to include the effect of free-free (bremsstrahlung) absorption. The latter is given by $P_{s}(x, z) \approx e^{-\tau_{\mathrm{ff}}(x, z)}$, where $\tau_{\mathrm{ff}}$ is the free-free absorption optical depth [46]. For $x \approx x_{0}$, photon absorption becomes relevant at redshift $z \gtrsim z_{\mathrm{abs}}=1700$.

Before proceeding, we comment on another possible signature from conversion at low redshifts, as the decay of $a$ inside clusters of galaxies will lead to a flux of $A^{\prime}$. The latter could be converted to regular photons as dark photons 

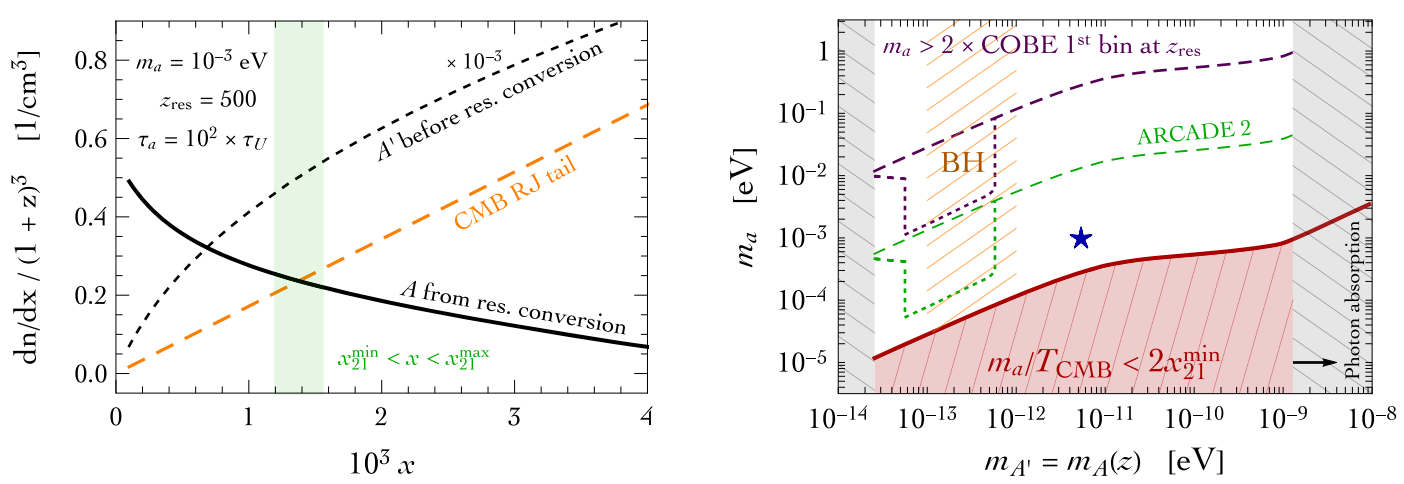

FIG. 3. (Left) (Dark) Photon spectrum (before) after conversion shown in solid black (dashed black, rescaled by $10^{-3}$ ) and compared to the CMB (orange) for $m_{a}=10^{-3} \mathrm{eV}, \tau_{a}=100 \times \tau_{U}, m_{A^{\prime}}=5 \times 10^{-12} \mathrm{eV}$ (implying resonance at $z_{\text {res }}=500$ ) and $\epsilon=2.1 \times 10^{-7}$. (Right) The relevant range of DM mass $m_{a}$ versus $m_{A^{\prime}}$ for addressing the $21 \mathrm{~cm}$ absorption signal; for $m_{a} / T_{\mathrm{CMB}}<2 x_{21}^{\min }$, the produced photons are too soft to impact $21 \mathrm{~cm}$. The region above the dashed purple (green) line are probed by FIRAS (ARCADE 2), constraining $\epsilon$ and $\tau_{a}$. The orange hatched region labelled as BH is possibly disfavored by black hole superradiance [48]. The star shows the example point considered in the left figure.

travel outside such a cluster, from high to low density. The typical density of electrons inside clusters, $n_{e} \sim 10^{-3} \mathrm{~cm}^{-3}$ [47], implies resonant conversions when $m_{A^{\prime}} \sim 10^{-12} \mathrm{eV}$. Thus, radio and microwave emission from clusters may contain new line components; this will be addressed separately.

Dark age resonance and EDGES signal.-For $z \leq z_{\mathrm{abs}}$, the Universe becomes transparent to photons that are converted into the RJ tail of the CMB, $x \sim 10^{-3}$, whereas for $z>z_{\mathrm{abs}}$, these soft photons are efficiently absorbed [46]. Therefore, only dark photons with $m_{A}<m_{A^{\prime}}\left(z_{\text {abs }}\right) \simeq$ $10^{-9} \mathrm{eV}$ will yield excess radiation at $21 \mathrm{~cm}$. Focusing on a monochromatic injection of $A^{\prime}$ with cosmologically long lifetime $\tau_{a}>\tau_{U}$, the energy spectrum at redshift $z$ reads

$$
\frac{d n_{A^{\prime}}}{d \omega}(\omega, z)=\frac{2 \Omega_{a} \rho_{c}(1+z)^{3}}{m_{a} \tau_{a} \omega H(\alpha-1)} \Theta(\alpha-1-z) .
$$

Here, $\rho_{c}$ is the critical density $\Omega_{a} h^{2}=0.12$, and the Hubble rate $H(z)$ is evaluated at redshift $\alpha-1$, where $\alpha \equiv m_{a}(1+z) /(2 \omega)$. The total number of injected $A^{\prime}$ grows with cosmic time $t(z)$, and can eventually outgrow $n_{\mathrm{CMB}}$ by a large margin, $n_{A^{\prime}}(z)=\left(6 \mathrm{eV} / m_{a}\right)\left[t(z) / \tau_{a}\right] \times n_{\mathrm{CMB}}(z)$.

Once the resonance condition is met at $z=z_{\text {res }}$, a fraction of $A^{\prime}$ will be converted as per Eq. (2). The final spectrum of converted photons at $z_{\text {res }}$ is given by Eq. (7) multiplied by the conversion probability of Eq. (6). To describe the spectrum at $z<z_{\text {res }}$, it is convenient to switch to redshift-independent $x=\omega / T_{\mathrm{CMB}}$, noting that $d n / d x$ scales as $(1+z)^{3}$ to account for the expansion of the Universe. The left panel of Fig. 3 shows the spectrum before and after conversion, in comparison to the RJ tail of the $\mathrm{CMB}$, assuming $m_{a}=10^{-3} \mathrm{eV}, \tau_{a}=100 \times \tau_{U}$, $z_{\text {res }}=500$, and $\epsilon=2.1 \times 10^{-7}$. For these parameters, the total energy density of dark photons relative to the energy density of ordinary CMB photons at resonance is $6 \times 10^{-6}$, and a fraction $4 \times 10^{-4}$ of the dark photons oscillate into ordinary photons.

In order to identify models that can be tested with $21 \mathrm{~cm}$ observations, we compare the number density of converted photons $n_{A^{\prime} \rightarrow A}$ to the RJ density of the CMB $n_{\mathrm{RJ}}$, within a relevant energy window. We define this window to include all photons with a wavelength of $21 \mathrm{~cm}$ within the redshift interval $z=15-20$. This is equivalent to requiring $x \in\left(x_{21}^{\min }, x_{21}^{\max }\right)=(1.2,1.6) \times 10^{-3}$.

The right panel of Fig. 3 shows the $\left\{m_{A^{\prime}}, m_{a}\right\}$ parameter space relevant for the $21 \mathrm{~cm}$ signal. Low values of $m_{a}$ give photons that are too soft to affect the $21 \mathrm{~cm}$ absorption line, while high values produce photons at energies that (depending on other parameters) could be probed by FIRAS and ARCADE 2. Low and high limiting values for $m_{A^{\prime}}$ originate from the requirement of a resonance in the relevant redshift window, with some interval $10^{-13}-10^{-12} \mathrm{eV}$ possibly disfavored by black hole superradiance [48] (see also [49,50]). However, it is worth noting that these bounds were derived for a massive free dark photon without including interactions due to the mixing term, and their rigorous applicability in our case deserves a dedicated analysis.

We determine by how much the RJ photon count can be increased due to $A^{\prime} \rightarrow A$ conversion in the $1.2 \times 10^{-3}<$ $x<1.6 \times 10^{-3}$ interval. The left panel of Fig. 4 shows the allowed values for the RJ photon increase, assuming $z_{\text {res }}=500$, when the lifetime $\tau_{a}$ of the DM particle is varied. We observe that the photon count can be increased by more than an order of magnitude with higher values limited by the stellar bound. If $n_{A^{\prime} \rightarrow A} / n_{\mathrm{RJ}}$ is kept constant, while $\tau_{a}$ is increased, the required value of $\epsilon$ is eventually excluded by CMB spectral distortions. Still, we find that the unexpected strength of the EDGES signal, which would require roughly $n_{A^{\prime} \rightarrow A} / n_{\mathrm{RJ}} \simeq 1$, is easily met over 4 orders of magnitude in the lifetime $\tau_{a} \sim\left(10-10^{5}\right) \tau_{U}$. Finally, the right panel of the same figure presents the $\left\{m_{A^{\prime}}, \epsilon\right\}$ parameter 

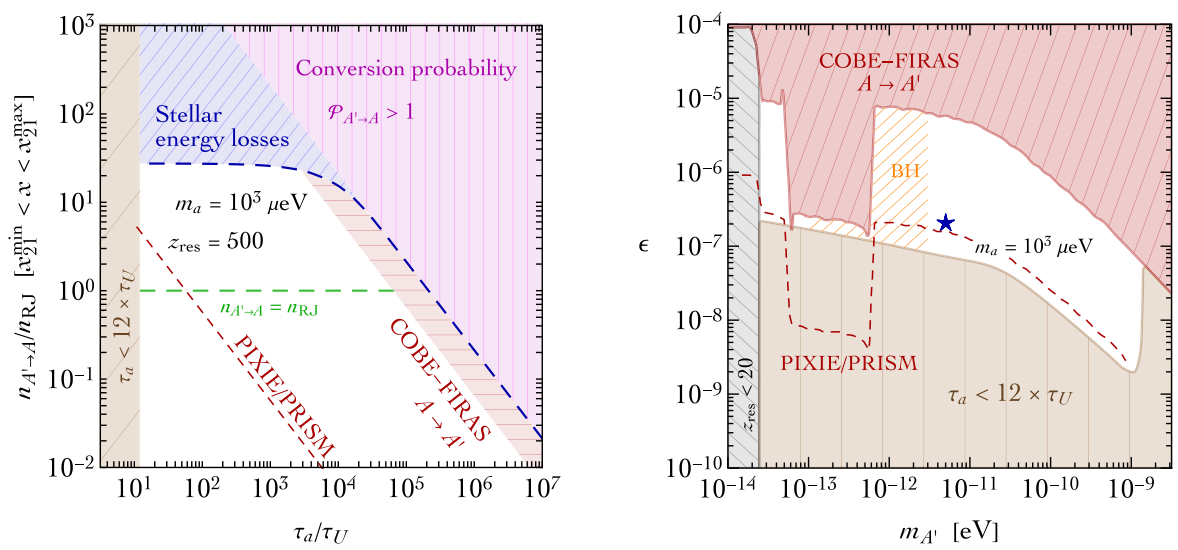

FIG. 4. (Left) Allowed region in DM lifetime versus number ratio of converted-to-original photons $n_{A^{\prime} \rightarrow A} / n_{\mathrm{RJ}}$, in the energy window $x_{21}^{\min }<x<x_{21}^{\max }$. The blue region is excluded by stellar energy loss; the purple region would require $P_{A^{\prime} \rightarrow A}>1$. (Right) Allowed parameter space in the $\left(m_{A^{\prime}}, \epsilon\right)$ plane; $\tau_{a}$ is chosen at each point such that $n_{A^{\prime} \rightarrow A} / n_{\mathrm{RJ}}=1$ in the $21 \mathrm{~cm}$ signal region. In both plots $m_{a}=10^{3} \mu \mathrm{eV}$, the brown region is excluded by a limit on the DM lifetime [12] and the red region (red dashed line) is excluded by FIRAS (would be probed by PIXIE/PRISM) due to $A \rightarrow A^{\prime}$ oscillations [15].

space, where $\tau_{a}$ is chosen such that $n_{A^{\prime} \rightarrow A} / n_{\mathrm{RJ}}$ is set to one. We find significant allowed parameter space, which can be mostly probed by the proposed PIXIE/PRISM experiments that are sensitive to spectral distortions [15].

Conclusions. - We have shown that the RJ tail of the CMB spectrum can be modified by light and weakly coupled new physics particles or fields without contradicting other cosmological or astrophysical constraints. We have presented one such example where the resonant conversion of nonthermal and numerous dark photons to ordinary photons leads to an enhancement in the RJ tail of the CMB. The upcoming era of precision $21 \mathrm{~cm}$ cosmology, as perhaps signaled by the first reported tentative detection [20], will open a window into such new physics.

We thank Yacine Ali-Haïmoud and Jens Chluba for helpful conversations. M. P. and J. T.R. acknowledge the financial support provided by CERN. Research at Perimeter Institute is supported by the Government of Canada through Industry Canada and by the Province of Ontario through the Ministry of Economic Development \& Innovation. J. P. is supported by the New Frontiers program of the Austrian Academy of Sciences. J. T. R. is supported by NSF CAREER Grant No. PHY-1554858.

[1] J. C. Mather et al., Astrophys. J. 420, 439 (1994).

[2] G. Hinshaw et al. (WMAP Collaboration), Astrophys. J. Suppl. Ser. 208, 19 (2013).

[3] P. A. R. Ade et al. (Planck Collaboration), Astron. Astrophys. 594, A13 (2016).

[4] K. N. Abazajian et al. (CMB-S4 Collaboration), arXiv:1610 .02743 .

[5] S. Furlanetto, S. P. Oh, and F. Briggs, Phys. Rep. 433, 181 (2006).
[6] J. Jaeckel and A. Ringwald, Annu. Rev. Nucl. Part. Sci. 60, 405 (2010).

[7] J. Alexander et al. arXiv:1608.08632.

[8] M. Battaglieri et al., arXiv:1707.04591.

[9] J. F. Cherry, M. T. Frandsen, and I. M. Shoemaker, Phys. Rev. Lett. 114, 231303 (2015).

[10] Y. Cui, M. Pospelov, and J. Pradler, Phys. Rev. D 97, 103004 (2018).

[11] Z. Berezhiani, A. D. Dolgov, and I. I. Tkachev, Phys. Rev. D 92, 061303 (2015).

[12] V. Poulin, P. D. Serpico, and J. Lesgourgues, J. Cosmol. Astropart. Phys. 08 (2016) 036.

[13] B. Holdom, Phys. Lett. B 166, 196 (1986).

[14] A. Mirizzi, J. Redondo, and G. Sigl, J. Cosmol. Astropart. Phys. 03 (2009) 026.

[15] K. E. Kunze and M. Vzquez-Mozo, J. Cosmol. Astropart. Phys. 12 (2015) 028.

[16] D. J. Fixsen, E. S. Cheng, J. M. Gales, J. C. Mather, R. A. Shafer, and E. L. Wright, Astrophys. J. 473, 576 (1996).

[17] A. E. Nelson and J. Scholtz, Phys. Rev. D 84, 103501 (2011).

[18] P. Arias, D. Cadamuro, M. Goodsell, J. Jaeckel, J. Redondo, and A. Ringwald, J. Cosmol. Astropart. Phys. 06 (2012) 013.

[19] P. W. Graham, J. Mardon, and S. Rajendran, Phys. Rev. D 93, 103520 (2016).

[20] J. D. Bowman, A. E. E. Rogers, R. A. Monsalve, T. J. Mozdzen, and N. Mahesh, Nature (London) 555, 67 (2018).

[21] M. Zaldarriaga, S. R. Furlanetto, and L. Hernquist, Astrophys. J. 608, 622 (2004).

[22] D. J. Fixsen et al., Astrophys. J. 734, 5 (2011).

[23] S. T. Staggs, N. C. Jarosik, D. T. Wilkinson, and E. J. Wollack, Astrophys. Lett. Commun. 32, 3 (1995).

[24] M. Bersanelli, G. F. Smoot, M. Bensadoun, G. de Amici, M. Limon, and S. Levin, Astrophys. Lett. Commun. 32, 7 (1995).

[25] H. Tashiro, K. Kadota, and J. Silk, Phys. Rev. D 90, 083522 (2014). 
[26] J. B. Muñoz, E. D. Kovetz, and Y. Ali-Haïmoud, Phys. Rev. D 92, 083528 (2015).

[27] R. Barkana, Nature (London) 555, 71 (2018).

[28] A. Ewall-Wice, T. C. Chang, J. Lazio, O. Dore, M. Seiffert, and R. A. Monsalve, arXiv:1803.01815.

[29] R. Barkana, N. J. Outmezguine, D. Redigolo, and T. Volansky, arXiv:1803.03091.

[30] A. Fialkov, R. Barkana, and A. Cohen, Phys. Rev. Lett. 121, 011101 (2018).

[31] S. Fraser et al., arXiv:1803.03245.

[32] C. Dvorkin, K. Blum, and M. Kamionkowski, Phys. Rev. D 89, 023519 (2014).

[33] V. Gluscevic and K. K. Boddy, arXiv:1712.07133.

[34] W. L. Xu, C. Dvorkin, and A. Chael, Phys. Rev. D 97, 103530 (2018).

[35] J. B. Muñoz and A. Loeb, Nature (London) 557, 684 (2018).

[36] A. Berlin, D. Hooper, G. Krnjaic, and S. D. McDermott, Phys. Rev. Lett. 121, 011102 (2018).

[37] G. D’Amico, P. Panci, and A. Strumia, Phys. Rev. Lett. 121, 011103 (2018).

[38] J. Bernstein, M. Ruderman, and G. Feinberg, Phys. Rev. 132, 1227 (1963).

[39] G. Raffelt and A. Weiss, Astron. Astrophys. 264, 536 (1992).
[40] M. Haft, G. Raffelt, and A. Weiss, Astrophys. J. 425, 222 (1994); 438, 1017(E) (1995).

[41] R. Essig et al., in Proceedings, 2013 Community Summer Study on the Future of U.S. Particle Physics: Snowmass on the Mississippi (CSS2013): Minneapolis, MN, 2013, http://www.slac.stanford.edu/econf/C1307292/ Snowmass2013Proceedings.html.

[42] H. An, M. Pospelov, and J. Pradler, Phys. Lett. B 725, 190 (2013).

[43] J. Redondo and G. Raffelt, J. Cosmol. Astropart. Phys. 08 (2013) 034.

[44] T.-K. Kuo and J. T. Pantaleone, Rev. Mod. Phys. 61, 937 (1989).

[45] S. J. Parke, Phys. Rev. Lett. 57, 1275 (1986).

[46] J. Chluba, Mon. Not. R. Astron. Soc. 454, 4182 (2015).

[47] N. A. Bahcall, arXiv:astro-ph/9611148.

[48] V. Cardoso, O. J. C. Dias, G. S. Hartnett, M. Middleton, P. Pani, and J. E. Santos, J. Cosmol. Astropart. Phys. 03 (2018) 043.

[49] P. Pani, V. Cardoso, L. Gualtieri, E. Berti, and A. Ishibashi, Phys. Rev. Lett. 109, 131102 (2012).

[50] M. Baryakhtar, R. Lasenby, and M. Teo, Phys. Rev. D 96, 035019 (2017). 